\title{
Sister Chromatid
}

National Cancer Institute

\section{Source}

National Cancer Institute. Sister Chromatid. NCI Thesaurus. Code C13455.

Sister chromatids are the two newly synthesized daughter chromatids derived from the same chromosome parent. During chromosomal pairing in meiosis, recombination occurs exclusively between non-sister chromatids. 